\title{
Review article: Practical current issues in perioperative patient safety
}

\section{Article de synthèse: Problèmes pratiques actuels pour la sécurité périopératoire des patients}

\author{
John H. Eichhorn, MD
}

Received: 26 June 2012/ Accepted: 27 November 2012/Published online: 20 December 2012

(c) Canadian Anesthesiologists' Society 2012

\begin{abstract}
Purpose This brief review provides an overview and, importantly, a context perspective of relevant current practical issues in perioperative patient safety.

Principal findings The dramatic improvement in anesthesia patient safety over the last 30 years was not initiated by electronic monitors but, rather, largely by a set of behaviours known as "safety monitoring" that were then made decidedly more effective by extending the human senses through electronic monitoring, for example, capnography and pulse oximetry. In the highly developed world, this current success is threatened by complacency and production pressure. In some areas of the developing/ underdeveloped world, the challenge is implementing the components of anesthesia practice that will bring safety improvements to parallel the overall current success, for instance, applying the World Federation of Societies of Anaesthesiologists (WFSA) "International Standards for A Safe Practice of Anaesthesia". Generally, expanding the current success in safety involves many practical issues. System issues involve research, effective reporting mechanisms and analysis/broadcasting of results, perioperative communication (including "speaking up to power"), and checklists. Monitoring issues involve enforcing existing published monitoring standards and also recognizing the risk of danger to the patient from hypoventilation during procedural sedation and from postoperative intravenous pain medications. Issues of clinical care include medication errors in the operating room, cerebral hypoperfusion
\end{abstract}

J. H. Eichhorn, MD (ه)

Department of Anesthesiology, College of Medicine, University

of Kentucky, N-202, UKMC, 800 Rose St., Lexington,

KY 40536-0293, USA

e-mail: jeichhorn@uky.edu (especially in the head-up position), dangers of airway management, postoperative residual weakness from muscle relaxants, operating room fires, and risks specific in obstetric anesthesia.

Conclusions Recognition of the issues outlined here and empowerment of all anesthesia professionals, from the most senior professors and administrators to the newest practitioners, should help maintain, solidify, and expand the improvements in anesthesia and perioperative patient safety.

\section{Résumé}

Objectif Cet article de synthèse court fournit une vue d'ensemble et surtout, une mise en contexte des problèmes pratiques actuels pertinents ayant trait à la sécurité périopératoire des patients.

Constatations principales L'impressionnante amelioration de la sécurité de l'anesthésie au cours des 30 dernières années n'a pas été déclenchée par les moniteurs électroniques, mais plutôt dans une large proportion, par un ensemble de comportements regroupés sous le terme "monitorage de la sécurité" "; ceux-ci ont été rendus nettement plus efficaces en elargissant la capacité sensorielle des humains grâce au monitorage electronique; la capnographie et l'oxymétrie de pouls en sont des exemples. Dans les pays hautement développés, ce succès actuel est menacé par un excès de confiance et la pression de la productivité. Dans quelques régions du monde en développement, le défi consiste à mettre en cuvve les eléments d'une pratique de l'anesthésie qui apporteront des améliorations en termes de sécurité pour parvenir au même niveau que dans le reste du monde en appliquant, par exemple, les "Normes internationales pour une pratique sécuritaire de l'anesthésie » de la Fédération internationale des sociétés d'anesthésiologistes, la WFSA (World Federation of Societies of Anaesthesiologists). D'une manière générale, l'extension du succès actuel en matière de 
sécurité implique de nombreux problèmes pratiques. Les difficultés liées au système impliquent de la recherche, des mécanismes efficaces de signalement des problèmes et une analyseldiffusion des résultats, une communication périopératoire (y compris un "dialogue avec les autorités » et des listes de contrôle. La question du monitorage implique l'application stricte des normes de monitorage existantes et publiées, ainsi que l'identification du risque de danger pour le patient lié à une hypoventilation au cours d'une sédation pour une intervention et aux médicaments antalgiques intraveineux administrés en postopératoire. Les problèmes liés aux soins médicaux incluent les erreurs de médicaments en salle d'opération, l'hypoperfusion cérébrale (en particulier quand le patient est dans une position avec la tête elevée), les dangers de la prise en charge des voies aériennes, la faiblesse musculaire résiduelle provoquée par les curares, les incendies en salle d'opération et les risques spécifiques à l'anesthésie obstétricale.

Conclusions L'identification des problèmes soulignés ici et le renforcement de l'autonomie de tous les professionnels de l'anesthésie, des professeurs et administrateurs les plus chevronnés jusqu'aux praticiens débutants, doivent aider à maintenir, renforcer et étendre les progrès de la sécuritédes patients sous anesthésie et en période périopératoire.

Enormous progress in anesthesia patient safety has occurred since the early 1980s. Principal practical current issues in anesthesia patient safety include 1) the preservation and further extension of the gains that have been attained from this progress in the highly developed world (explained below) and 2) the facilitation, encouragement, and adoption of all underlying progress-promoting components of practice in the relevant areas of the developing and underdeveloped world.

Due to the absence of comprehensive statistics, discovery of genuine cases, and consistent definitions of anesthesia-related morbidity and mortality, there has been and will be debate regarding the specific details of the remarkable improvement in anesthesia patient safety. However, there can be no question of this dramatic advance, particularly for intraoperative catastrophic accidents involving death, brain damage, and cardiac arrest. While classic " $P<0.05$ " statistical "proof" with fully controlled randomized prospective trials likely will never be possible (analogous to the discussion of the efficacy of parachutes), ${ }^{1}$ the experience of the reduction in malpractice insurance premiums, specifically for American anesthesiologists, ${ }^{2,3}$ provides an "alternate definition of truth" that substantiates an extraordinary reduction in the number and severity of patient injuries caused solely by anesthesia care accidents. Contrary to popular impressions, new technology was not the principal factor behind improved anesthesia patient safety. The main initiator of change was the codification and institutionalization of a set of behaviours in clinical anesthesia care that are symbolized by the protocols and related standards for "safety monitoring" 4 and involve a genuine and continuous increase in intraoperative vigilance. Of course, these behaviours were also enormously empowered by the extension of the human senses through electronic monitors, particularly the capnograph and pulse oximeter. At the same time, there were many other factors that contributed to safer care, including improvements in medications, quality of trainees, and training programs.

While very logical and desirable, preserving the gains in anesthesia patient safety in the developed world is not guaranteed. First, there is a significant paradoxical, even ironic, threat caused specifically by the remarkable success in making anesthesia safer - complacency by anesthesia professionals. Essentially, anesthesia care has become so safe that a great many modern anesthesia professionals were trained and have practiced in an era when anesthesia catastrophes, such as death from an unrecognized esophageal intubation, are vanishingly rare. Thus, many current practitioners not only have never experienced a major accident, but also have never seen or even heard of one. It is hoped that highly experienced practitioners who trained before safety was improved still practice with a healthy respect for and even fear of the consequences of lapses in intraoperative vigilance - rather than falling into a false confidence that catastrophic accidents can no longer happen. Less experienced practitioners are so familiar with the modern behaviours and technologies that they often think they have always existed. They should be continually reminded to take nothing for granted because administering anesthesia involves inherent potential danger to the patient. Further, and even more concerning for all anesthesia professionals, is the current cultural-economic climate in which more clinical service is expected but with fewer resources (people and finances) to provide it. This issue causes "production pressure" in clinical anesthesia care that translates into "go faster and do it for less cost". The inevitable temptation to cut corners and employ inherently unsafe practices may temporarily slightly increase efficiency and reduce cost. Ultimately, it will contribute to a classic "Swiss cheese" situation" when the "holes line up" as multiple deviations from normal practice coincide in time to allow a catastrophic accident.

In some areas of developing/underdeveloped locations, the issues are often more basic. Recognition of trained anesthesia professionals as a necessary and fully equal component of surgical care is not universal. Governmental and private healthcare systems as well as facility administrators must be educated and guided on this point 
wherever necessary. This was a key component of the supporting documents for the original "International Standards for a Safe Practice of Anaesthesia" created by the ad hoc International Task Force on Anaesthesia Safety and first adopted as world standards in 1992 by the WFSA. ${ }^{6}$ In addition, recognition of the variation in availability of resources led to division of the prescribed "required" facilities and equipment for anesthesia care into three levels: basic, intermediate, and optimal. All of those principles and additional related ones persist in the recently updated version of the WFSA standards. ${ }^{7,8}$ Likewise, as part of the World Health Organization (WHO) "Safe Surgery Saves Lives" global campaign that features the well-known "Surgical Safety Checklist", 9 the WHO Safe Anesthesia Working Group expanded the supporting technical documents to include, in particular, an extensive presentation of a "Guide to infrastructure, supplies, and anaesthesia standards at three levels of healthcare facilities". ${ }^{10}$ While these and similar documents apply to all anesthetizing locations around the globe, they are especially valuable in outlining a detailed proven approach to the practical current issue of encouraging and facilitating anesthesia patient safety efforts in the relevant areas of the developing/underdeveloped world.

\section{System issues}

A significant current issue is the lack of organized systems of classification, reporting, and study of elements of patient safety, both for anesthesia care and for the healthcare establishment in general.

The WHO project, The International Patient Safety Classification, aims "to define, harmonize, and group patient safety concepts into an internationally agreed classification" in order to promote study and improvement of safety. It will serve the profession well to have an organized taxonomy for patient safety efforts, whether through this mechanism or through a similarly tasked alternate. A difficult feature, for example, will be defining an "anesthesia-related death" for statistical purposes. There is a precedent in the Data Dictionary Task Force sponsored by the Anesthesia Patient Safety Foundation (APSF) in the U.S. This task force is a consortium of anesthesiologists and manufacturers of anesthesia information management systems (i.e., automated electronic anesthesia records) that has assembled and hammered out accepted definitions for anesthesia-related terminology so that it is consistent when adopted in various brands of electronic anesthesia records and, thus, can be used for multicentre data gathering, analysis, and quality enhancement. ${ }^{11}$ A parallel effort in anesthesia safety would be significantly beneficial to facilitate the continued progress of safety improvement.

It has long been a goal of experts in the field to create comprehensive reporting systems for anesthesia-related adverse events, complications, and critical incidents that would then allow analysis and pattern recognition. The benefit of identifying evolving trends in adverse events is two-fold. First, once a problem is identified, anesthesia professionals can quickly be warned of the danger in an effort to reduce or eliminate the events. Second, and closely related, is the intent to analyze the reports thoroughly in order to develop "best practices" recommendations that can be both publicized and circulated to the anesthesia community and also studied to document their efficacy in improving anesthesia patient safety. One very focused example of such an effort is the Postoperative Vision Loss Registry of the American Society of Anesthesiologists (ASA). ${ }^{12}$ Also in the U.S., the ASA has established the Anesthesia Quality Institute ${ }^{13}$ with its Anesthesia Incident Reporting System. ${ }^{14}$ It was established to serve, in part, as a repository of outcome information from anesthesia practices with the intent to build a large database that can be used by safety researchers who study practice patterns in order to identify clinical dangers and propose ways to mitigate them. Various reporting systems for adverse events in anesthesia care have been proposed, including alliances of professional societies and governments. One current effective model is The Australian and New Zealand Tripartite Anaesthetic Data Committee, ${ }^{15}$ which is a revival of the original Australian Incident Monitoring System ${ }^{16}$ that, for many years, set the example for reporting adverse events. In all cases, concerns about confidentiality/anonymity and potential medical-legal implications of honestly reporting complications that cause patient injury are paramount and have often slowed or halted implementation of such potentially valuable systems. The WHO has published a generic guide for reporting adverse events ${ }^{17}$ that can help support efforts to create such mechanisms.

With defined terms and data to work with, anesthesia researchers in patient safety should be able to identify patterns of adverse events and analyze them with the intent of improving safety. Although the number of successful projects has been limited, one outstanding example of this strategy is the ASA's Closed Claims Study. ${ }^{18}$ Since its creation in 1985, this effort has been very productive in helping improve anesthesia care through establishing and building a database and then identifying and analyzing complications. This endeavour has led to several "best practices" type recommendations from the ASA. Human factor engineers and human error experts working with anesthesiologists have published analyses of safety problems, such as the implications of the ever-increasing intensification of technology in clinical care. ${ }^{19}$ These types 
of studies illustrate this relevant practical current issue (and challenge), i.e., the profession needs to develop and utilize all these systems to "translate" from theoretical to practical applications in front-line clinical care both to help maintain the gains in anesthesia patient safety and also to extend them to the next generation of further improvements.

Communication as a component of anesthesia care is another practical issue with major safety implications. Of importance are the several communication elements among members of the operating room team in the WHO Surgical Safety Checklist; all elements are intended to smooth the surgical process and to help prevent errors that could lead to injury. Obviously, inadequate or faulty recording and transmission of key preoperative evaluation data can create risks to patients. This may be especially likely in an atmosphere of high pressure to produce, where the facility and its administrators value speed over accurate completeness - until there is an injury accident that costs far more than many years' worth of increased profits from an accelerated workflow. Handoffs of care between two anesthesia professionals depend on communication, and this daily occurrence has been studied with various results. However apparently logical, it is still being evaluated whether a structured system or protocol (such as a printed checklist) either on paper or on screen improves the process of anesthesia care and, specifically, the outcomes.

Another critical element of communication with significant safety implications is known as "speaking up to power". The concept involves preventing a situation where one member of a perioperative team recognizes a clear and present danger or a potential danger to a patient but is inhibited from calling attention to the danger because of a sociocultural inequality, intimidation, fear of reprisal (such as job loss), or simply being ignored. Again, however logical it may be that such situations are completely unacceptable, they do exist. When a danger is recognized by one member of a team, the "P.A.C.E." model of graded verbal assertiveness (originating in the commercial aviation industry) is an excellent protocol. It involves: Probe ("Do you see that..."), Alert ("Can we re-evaluate the..."), Challenge ("Please stop a moment and discuss..."), and Emergency ("STOP NOW!"). This concept is a valuable tool that should be taught to all anesthesia professionals. Good role models are needed as teachers and leaders, and they also must support and back up their junior colleagues who do speak up to deal with or prevent dangerous situations. There is also an analogy to "lean manufacturing" where any worker in any position is permitted and, in fact, encouraged to "stop the line" or halt production by an entire factory when something is wrong and the quality of the product is threatened. Likewise, when a danger is seen, anyone in the perioperative environment must be empowered to speak up and even to stop the workflow in the interest of patient safety.

Finally, a prototypical systems issue is the value of checklists as applied to the process of anesthesia care. Use of the WHO Surgical Safety Checklist, which includes some anesthesia elements, has been shown to reduce surgical deaths and complications in diverse adult populations. ${ }^{20}$ It seems that the anesthesia community is advocating increasingly for the value of developing and implanting checklists to enhance anesthesia safety. One version of a proposed specific "anesthesia patient safety checklist" is shown in the Table.

\section{Monitoring issues}

An important practical current issue in anesthesia safety has two related aspects regarding the actual conduct of anesthetics: 1) In practice settings where basic monitoring standards/guidelines (such as those of the $\mathrm{ASA}^{21}$ and the Canadian Anesthesiologists' Society ${ }^{22}$ have been acknowledged and adopted, the standards must be followed for each and every applicable anesthetic. Logical as that may seem, there have been both anecdotal and occasional media reports of patients suffering anesthetic-related injuries that seem related to failure to monitor according to the standards. In settings with appropriate available resources, such preventable accidents are simply unacceptable. 2) In practice settings where monitoring standards/guidelines have not been adopted, the involved anesthesia professionals, ministries of health, and facility administrators should urge the necessary allocation of resources to make this happen as soon as possible. The "WFSA International Standards for a Safe Practice of Anesthesia 2010"7 is an excellent model of the goal of this effort, and the WFSA as an organization offers encouragement and support to any anesthesia professionals anywhere seeking help to improve patient safety. Likewise, Lifebox ${ }^{23}$ is an international non-profit charitable organization whose mission is "saving lives by improving the safety and quality of surgical care in low-resource countries" with a current goal of providing a pulse oximeter for every anesthetizing location in the world. Lifebox is an extension of the Global Oximetry Project ${ }^{24}$ and related efforts by WHO, all of which show resolve to promote patient safety protocols and improve anesthesia care throughout the world.

A more disturbing issue related to intraoperative monitoring is the fact that various anesthesiology societies and also regulatory bodies occasionally still receive questions or reports regarding anesthesia professionals leaving an anesthetized patient unattended in an operating room (OR) during surgery (with no remote monitoring or substitute 
Table Anesthesia patient safety checklist

1. VERIFY health and function of anesthesia professional(s): hand hygiene, available sanitizer, gloves; plan for OR noise control.

2. VERIFY appropriate anesthesia machine and breathing circuit checks (especially $100 \% \mathrm{O}_{2}$ deliverable by positive pressure) are performed successfully, including $\mathrm{CO}_{2}$ absorbent integrity.

3. VERIFY availability of devices: suction, separate self-inflating breathing bag, indicated warmers and humidifiers, neuromuscular stimulator (and plan for use prior to extubation).

4. VERIFY airway evaluation, airway tools/equipment (routine and, if indicated, special).

5. VERIFY monitors placed and working, including test of $\mathrm{CO}_{2}$ waveform, alarms set and on, vital signs (i.e., baseline and management goals).

6. VERIFY planned medications: availability, correct labelling, possible interactions, and emergency drugs.

7. VERIFY function of intravenous/vascular access or plan for insertion: fluids planned, available; infusion equipment.

8. CONSIDER implications of patient positioning, including pressure points, eyes, organ perfusion.

9. PERFORM “Timeout” functions per facility protocols, including at least:

a. Check patient identity and surgical site marking.

b. Drug allergies.

c. Anesthesia implications of co-existing conditions, current medications, lab and study results.

d. Nil per os (NPO) status, aspiration risk and planned countermeasures.

e. Fire risk.

f. Prediction of blood loss and, as indicated, blood availability.

g. Antibiotic and deep vein thrombosis (DVT) prophylaxis as indicated.

h. Discussion with team: projected case length, surgeon concerns, and potential complications.

person involved). The potential for a catastrophic accident is obvious, but this type of occurrence likely represents the psychological principle of the "get away with it phenomenon" in which an inherently unsafe action is repeated often enough without any adverse consequence to extinguish the appropriate concern/fear for the probable/ inevitable accident (as an example, seen regularly with texting while driving). Thus, it should be the responsibility of all anesthesia professionals to be aware of any such inappropriate behaviour by their colleagues and to counsel against it accordingly.

A significant and persistent current issue is patient safety problems related to monitoring patients who are receiving "sedation" for procedures or narcotic postoperative pain medication. The dangers of depressants causing unrecognized hypoventilation are well documented. In the operating room or in equivalent anesthetizing locations, potential situations often involve anesthesia professionals supervising patients who are receiving monitored anesthesia care with intravenous sedation or with regional anesthesia along with intravenous sedation supplementation. In that setting, the watchful eye of an experienced clinician paying close attention to the patient's breathing should help prevent dangerous hypoventilation. Likewise, the OR setting is more likely to have a capnograph that can be connected to a cannula in order to sample the patient's exhaled breath and give at least a "qualitative" indication of ongoing ventilation. The value of this strategy has been debated but, so far, one conclusion seems to be that this approach is better than nothing. One excellent comprehensive interdisciplinary set of guidelines for procedural sedation analgesia has been produced in Australia and New Zealand and was published in 2010 as a "professional standard". ${ }^{25}$ The guidelines stipulate that a dedicated practitioner should be assigned exclusive responsibility for administering the sedation and monitoring the patient, and the guidelines also state that capnography should be accessible within the facility. There have been suggestions that "qualitative capnography" should be employed in all "sedation" settings, such as gastrointestinal endoscopy, radiology, cardiac catheterization labs, as well as plastic surgery, dental/oral surgery, or other such suites/offices where intravenous hypnotics and narcotics are used to facilitate procedures. This has not occurred to any significant extent, largely because of unfamiliarity with the technology of capnography and also because of its potential costs. Thus, in many, if not most, settings where non-anesthesia practitioners administer intravenous sedation, patients are monitored with only a pulse oximeter as a "surrogate" ventilation monitor. Unfortunately, in many of these situations, the patients also receive supplemental oxygen by nasal cannulae. This significantly reduces the value of using pulse oximetry for monitoring ventilation because $\mathrm{SpO}_{2}$ is misleadingly maintained for extended periods of inadequate ventilation. Accident investigations of injuries in this situation frequently yield the declaration: "The patient was agitated, struggling, and moving around, so I gave more sedation. I increased the $\mathrm{O}_{2}$ flow when the saturation fell to $90 \%$; the patient's saturation came up to $99 \%$, but then she arrested ...." Improved education of those who administer sedation as well as protocols/guidelines provided by anesthesia professionals should help avoid these hypoventilation accidents. 
Unrecognized danger of hypoventilation from postoperative pain medication can be even more problematic because many patients are monitored only by intermittent observation and periodic measurements of vital signs. This issue has received considerable attention, ${ }^{26-29}$ and an appreciable number of preventable catastrophic accidents continue to occur. However, while increased monitoring of at-risk patients (particularly when patient-controlled analgesia is used) seems indicated, consensus has not been achieved regarding a "get to zero" approach of eliminating these mishaps. Again, concerned anesthesia professionals are encouraged to speak up in their practice settings, offer protocols and guidelines, and promote increased education of all involved practitioners.

\section{Clinical care issues}

Operating room medication errors continue to be of concern. Organized efforts have been offered by patient safety researchers, technology manufacturers, and safety organizations to help address these problems. The APSF published recommendations resulting from a U.S. national medication safety conference that focused on integrated approaches involving "standardization, technology, pharmacy/prefilled/premixed, and culture" 30 intended to stimulate discussion and action towards eliminating anesthesia medication errors.

Cerebral ischemic injuries from relative hypotension have sparked a lively debate about the adequacy of cerebral perfusion pressure during general anesthesia, particularly in the "head-up" or beach-chair position, which has been used increasingly in the last decade, for example, in arthroscopic surgery on the shoulder. Published analyses recognize the phenomenon, appreciate that there are differences of opinion on the physiology and monitoring of the cerebral circulation, and urge caution during these anesthetics. ${ }^{31}$ The APSF presented a national participatory workshop on the topic, ${ }^{32}$ and recommendations were drafted regarding the additional research required, the steps technology manufacturers can do to help solve the problem, the best practices for blood pressure management for these cases, and the additional measures the APSF can take to focus attention and action on these adverse outcomes.

Perioperative infections have been recognized only fairly recently as potentially involving anesthesia professionals as well as the surgeons, technicians, and nurses who work during surgery south of the proverbial "blood-brain barrier". Verification of the administration of indicated perioperative antibiotics in a timely manner has been incorporated into the "time-out" ritual in many operating suites. More directly, the focus on central venous catheters as the potential source of infection has led to the widespread adoption of "full-barrier" precautions with whole body draping of the patient and full gowning of all people involved in inserting the catheter. Likewise, all intravenous lines are potential portals for the introduction of infections, and there has been increased emphasis on hand hygiene for anesthesia professionals and aseptic techniques when using stopcocks or injection ports to administer intravenous medications.

Certainly, danger relating to airway management persists as a practical current issue. Analysis of the ASA Closed Claims Study ${ }^{18}$ shows airway management, including difficult tracheal intubation, as the most common cause of anesthesia-related patient injury since 1990 . While improved airway tools clearly help anesthesia professionals "bail out" patients when an unexpected difficult airway is encountered, the ability to predict difficult intubation preoperatively has not progressed to a parallel degree. It remains thought-provoking that anesthesia practitioners routinely fully paralyze patients without knowing whether or not they can intubate. This is not necessarily a failing, but merely a reality. Routine airway assessment does not identify all difficult airways. ${ }^{33}$ Nonetheless, consideration of this potential danger is always important, and the WHO Surgical Safety Checklist ${ }^{9}$ does include "difficult airway" as a question as well as "equipment/assistance available". Immediate availability of alternate airway devices, including video and fiber optic instruments as well as supraglottic airways, is critical, but it seems that increased research efforts into prediction of and planning for a difficult airway would help prevent both emergent need for bailout and risk of injury for the patient. Also, in a patient who initially required special effort for tracheal intubation, it was shown that risk of injury was associated with poor planning for airway management following tracheal extubation of the difficult airway. Extended close ventilation monitoring, potentially even in an intensive care unit, may be indicated to help the patient over the hump of the immediate postanesthesia period when airway swelling and secretions may be problematic.

Further examples of clinical care concerns that deserve recognition as current practical issues in anesthesia patient safety include: 1) Weakness from residual neuromuscular blockade that results in ventilatory distress following tracheal extubation at the end of an anesthetic. ${ }^{34,35}$ Arguments have been made for increased use of peripheral nerve stimulators to guide appropriate antagonism of nondepolarizing neuromuscular block and extubation decisions. This should come with increased primary and remedial education of anesthesia professionals on the precise application of the involved physiologic principles. 2) Operating room fires causing patient injury have recently received significant attention. This includes both airway fires and, especially, fires involving cloth or paper on the patient being ignited by electrocautery that was mistakenly 
used in an oxygen-rich environment (usually during monitored anesthesia care or sedation). ${ }^{36}$ The primary strategy for eliminating this danger is awareness and education of anesthesia professionals administering the oxygen as well as all operating room personnel regarding the risks and prevention of fire. 3) Obstetric (OB) anesthesia ${ }^{37}$ persists as a source of patient safety concerns. One issue often overlooked is excessive delay (sometimes caused by the obstetrician and/or the anesthesia provider failing to communicate) in commencing anesthesia for a stat Cesarean delivery for severe fetal distress. While this could be seen as an administrative matter, any resulting injury is just as real. Where this could be an issue, anesthesia practice organizers must always keep this point in their sights. When stat general anesthesia is induced for delivery, difficult tracheal intubation (sometimes caused by obesity and/or by the anatomic changes of term pregnancy) that causes hypoxemia threatens two patients. Analysis of injuries from such circumstances often suggests inappropriate persistence with direct laryngoscopy when earlier transition to alternate airway techniques may have helped avoid the hypoxic damage. When neuraxial anesthesia is employed for any OB patient, potential danger from an unrecognized high block that compromises ventilation should always be kept in mind. One of the most common OB safety risks due to delay is inadequate management of peripartum hemorrhage. Focus on and early recognition and treatment of excessive blood loss is the most effective strategy to prevent injury to both patients.

\section{Conclusions}

This brief review of practical current issues in anesthesia patient safety is by no means exhaustive, but a broad list of the most prominent concerns is offered with the intent to provoke consideration and action by all professionals involved, from the most senior professors and administrators to the newest front-line practitioners.

Many of the topics introduced here are explored in greater detail in subsequent review articles in this issue of the Journal, illustrating their importance in the overall effort. Those reviews provide more background information, analysis, and recommendations for promoting, solidifying, and expanding the current success in improving anesthesia safety.

Overall, anesthesia care is significantly safer than it was 30 years ago. The challenges today are to maintain that improvement in the face of pressures threatening the gains, to extend that improvement to additional aspects of anesthesia care as described above, and to help install the practice components shown to improve anesthesia patient safety in the areas of the world yet to see this benefit.

\section{Key points}

- Dramatic improvements in anesthesia patient safety began 30 years ago with the behaviours of "safety monitoring" facilitated by the introduction of widespread capnography and pulse oximetry.

- These improvements are threatened by complacency and production pressure. Further, these improvements have not penetrated some areas of the developing/ underdeveloped world, and efforts are needed to install the necessary education and resources to facilitate progress in patient safety.

- Understanding and action regarding several practical current issues can further expand improvements in anesthesia safety.

- System issues include data gathering and reporting, multiple aspects of communication, and checklists.

- Monitoring issues include enforcement of monitoring standards and understanding the risk of danger to the patient during sedation or owing to intravenous pain medications.

- Clinical care issues include medication errors in the operating room, cerebral hypoperfusion, airway management dangers, postoperative muscle weakness, operating room fires, and specific obstetric anesthesia dangers. Several of these topics are covered in additional detail in other reviews in this issue of the Journal.

Disclosure No financial or competing interests.

\section{References}

1. Smith GC, Pell JP. Parachute use to prevent death and major trauma related to gravitational challenge: systematic review of randomised controlled trials. BMJ 2003; 327: 1459-61.

2. Eichhorn JH. Monitoring standards: role of monitoring in reducing risk of anesthesia. In: Fleisher LA, Prough DS, editors. Problems in Anesthesia: Vol 13: Quality, Safety, Risk, and Outcomes. Philadelphia: Lippincott Williams \& Wilkins; 2001. p. 430-43.

3. Hallinan JT. Once Seen as Risky, One Group of Doctors Changes Its Ways: Anesthesiologists Now Offer Model of How to Improve Safety, Lower Premiums. Wall Street Journal. June 21, 2005, page A1. Available from URL: http://www.saynotocaps. org/newsarticles/once_seen_as_risky.htm (accessed June 20, 2012).

4. Eichhorn JH. Prevention of intraoperative anesthesia accidents and related severe injury through safety monitoring. Anesthesiology 1989; 70: 572-7.

5. Reason J. Human error: models and management. BMJ 2000; 320: 768-70.

6. International Taskforce on Anaesthesia Safety. Eur J Anaesthesiol 1993; 10(Supplement 7): 1-44.

7. Merry AF, Cooper JB, Soyannwo O, Wilson IH, Eichhorn JH. International Standards for a Safe Practice of Anesthesia 2010. Can J Anesth 2010; 57: 1027-34. 
8. Merry AF, Cooper JB, Soyannwo O, Wilson IH, Eichhorn JH. An iterative process of global quality improvement: the International Standards for a Safe Practice of Anesthesia 2010. Can J Anesth 2010; 57: 1021-6.

9. World Alliance for Patient Safety. World Health Organization. WHO Surgical Safety Checklist and Implementation Manual. Available from URL: http://www.who.int/patientsafety/safe surgery/ss_checklist/en/ (accessed June 20, 2012).

10. World Health Organization. World Alliance for Patient Safety. WHO Guidelines for Safe Surgery. Geneva: World Health Organization, 2008: 27-44. Available from URL: http://www.who. int/patientsafety/information_centre/reports/Alliance_Forward_ Programme_2008.pdf (accessed August 2012).

11. Anesthesia Patient Safety Foundation (APSF). Data Dictionary Task Force. Available from URL: http://www.apsf.org/initiatives_data.php (accessed June 21, 2012).

12. Lee LA, Roth S, Posner KL, et al. The American Society of Anesthesiologists Postoperative Visual Loss Registry: analysis of 93 spine surgery cases with postoperative visual loss. Anesthesiology 2006; 105: 652-9.

13. Anesthesia Quality Institute. Home of the National Anesthesia Clinical Outcomes Registry (NACOR). Available from URL: http://www.aqihq.org/ (accessed June 21, 2012).

14. Dutton RP. Introducing the Anesthesia Incident Reporting System (AIRS). American Society of Anesthesiologists 2011; 75: 30-3. Available from URL: http://www.aqihq.org/files/AIRS\% 20article\%200ct\%20ASA\%20newsletter.pdf (accessed June 21, 2012).

15. Australian and New Zealand College of Anaesthetists. Quality and Safety. Australian and New Zealand Tripartite Anaesthetic Data Committee. Available from URL: http://www.anzca.edu. au/fellows/quality-safety/australian-and-new-zealand-tripartiteanaesthetic-data-committee (accessed June 21, 2012).

16. Runciman WB, Webb RK, Lee $R$, Holland $R$. The Australian Incident Monitoring Study. System failure: an analysis of 2000 incident reports. Anaesth Intensive Care 1993; 21: 684-95.

17. World Health Organization. World Alliance for Patient Safety. WHO Draft Guidelines for Adverse Event Reporting and Learning Systems: From information to Action. Geneva: WHO, 2005. Available from URL: http://www.who.int/patientsafety/ events/05/Reporting_Guidelines.pdf (accessed August 2012).

18. Metzner J, Posner KL, Lam MS, Domino KB. Closed claims analysis. Best Pract Res Clin Anaesthesiol 2011; 25: 263-76.

19. Webster CS, Stabile M, Merry AF. The challenges of technological intensification. Anesthesia Patient Safety Foundation Newsletter 2009; 24: 33-44.

20. Haynes AB, Weiser TG, Berry WR, et al. A surgical safety checklist to reduce morbidity and mortality in a global population. N Engl J Med 2009; 360: 491-9.

21. American Society of Anesthesiologists. Standards, Guidelines, Statements and Other Documents. Available from URL: http:// www.asahq.org/For-Members/Standards-Guidelines-and-Statements. aspx (Basic Anesthetic Monitoring, Standards for (effective July 1, 2011) (accessed June 22, 2012).

22. Merchant $R$, Chartrand D, Dain S, et al. Guidelines to the Practice of Anesthesia - Revised Edition 2012. Can J Anesth 2012; 59: 63-102.
23. Lifebox Foundation. Saving Lives Through Safer Surgery. Available from URL: http://www.lifebox.org (accessed June 22, 2012).

24. Merry AF, Eichhorn JH, Wilson IH. Extending the WHO 'Safe Surgery Saves Lives' project through global oximetry. Anaesthesia 2009; 64: 1045-8.

25. Australian and New Zealand College of Anaesthetists; Faculty of Pain Medicine Australian and New Zealand College of Anaesthetists; Gastroenterological Society of Australia; Royal Australasian College of Surgeons; Australasian College for Emergency Medicine; College of Intensive Care Medicine of Australia and New Zealand; Royal Australasian College of Dental Surgeons; Royal Australian and New Zealand College of Radiologists. Guidelines on Sedation and/or Analgesia for Diagnostic and Interventional Medical, Dental or Surgical Procedures - 2010. Available from URL: http://www.anzca.edu.au/ resources/professional-documents/documents/professional-standards/ pdf-files/PS9-2010.pdf (accessed July 31, 2012).

26. Weinger MB. Dangers of postoperative opioids: APSF workshop and white paper address prevention of postoperative respiratory complications. APSF Newsletter 2006-2007; 21: 61-7.

27. Dahan A, Aarts L, Smith TW. Incidence, reversal, and prevention of opioid-induced respiratory depression. Anesthesiology 2010; 112: 226-38.

28. Weinger MB, Lee LA. No patient shall be harmed by opioidinduced respiratory depression. APSF Newsletter 2011; 26: 21, 26-8.

29. Taenzer A, Blike G. Postoperative monitoring - the Dartmouth experience. APSF Newsletter 2012; 27: 1, 3-4, 21.

30. Eichhorn JH. APSF hosts medication safety conference: Consensus group defines challenges and opportunities for improved practice. APSF Newsletter 2010; 25: 1, 3-8.

31. Lanier WL. Cerebral perfusion: err on the side of caution. APSF Newsletter 2009; 24: 3-11.

32. Lee L, Caplan R. APSF Workshop: Cerebral perfusion experts share views on management of head-up cases. APSF Newsletter 2009-2010; 24: 45, 47-8.

33. Shiga T, Wajima Z, Inoue T, Sakamoto A. Predicting difficult intubation in apparently normal patients: a meta-analysis of bedside screening test performance. Anesthesiology 2005; 103: 429-37.

34. Murphy GS, Szokol JW, Marymont JH, Greenberg SB, Avram MJ, Vender JS. Residual neuromuscular blockade and critical respiratory events in the postanesthesia care unit. Anesth Analg 2008; 107: $130-7$

35. Marcus A. Residual paralysis: the problem that won't go away. Anesthesiology News 2012; 38: 1. Available from URL: http:// www.anesthesiologynews.com/ViewArticle.aspx?d=Clinical\% 2BAnesthesiology\&d_id=1\&i=July $+2012 \& i \_i d=864 \& a \_i d=$ 21217 (accessed July 31, 2012).

36. Anesthesia Patient Safety Foundation (APSF). Fire Safety Video. Prevention and Management of Operating Room Fires, 2010. Available from URL: http://www.apsf.org/resources_video.php (accessed June 25, 2012).

37. Ross BK. ASA closed claims in obstetrics: lessons learned. Anesthesiol Clin North America 2003; 21: 183-97. 